\title{
Investigation of the Fragmentation Process of Clods in a Rod Mill Developed for Research Use
}

\author{
Masayuki Oishi' ${ }^{1}$ Yoshihiro Kubota2 ${ }^{2 *}$, Osamu Mochizuki ${ }^{3}$ \\ ${ }^{1}$ Daiki Rika Kogyo Co. Ltd., Saitama, Japan \\ ${ }^{2}$ Department of Mechanical Engineering, Toyo University, Saitama, Japan \\ ${ }^{3}$ Department of Biomedical Engineering, Toyo University, Saitama, Japan \\ Email: ^kubota548@toyo.jp
}

How to cite this paper: Oishi, M., Kubota Y. and Mochizuki, O. (2019) Investigation of the Fragmentation Process of Clods in a Rod Mill Developed for Research Use. World Journal of Mechanics, 9, 233-243. https://doi.org/10.4236/wjm.2019.910015

Received: September 18, 2019

Accepted: October 7, 2019

Published: October 10, 2019

Copyright (c) 2019 by author(s) and Scientific Research Publishing Inc. This work is licensed under the Creative Commons Attribution International License (CC BY 4.0).

http://creativecommons.org/licenses/by/4.0/ (c) (i) Open Access

\begin{abstract}
We developed a planetary-type rod mill that can improve the comminution working efficiency, prevent the generation of soil dust, and make it easy to clean the used jars. Clods are broken by this device into fine-grained soil particles with sizes of less than $2 \mathrm{~mm}$ in diameter within $2 \mathrm{~min}$. One objective of this study is to evaluate the performance of the mill by observing the fragmentation process of the clods. The process is visualized experimentally via a high-speed camera installed on a rotating jar of the device. The breakage of the clods in the rod mill is considered morphologically according to the crushing patterns obtained by previous studies. The rod moves together with the clods in the jar. Therefore, violent collisions are not thought to be a major reason for the breakage of the clods. The surfaces of the clods are first scraped and then appear to become fragile. However, if there are no rods in the jar, the clods never break into small pieces. Therefore, it is likely that collisions with the rod trigger the catastrophic fragmentation of the clods into small pieces. This suggests that the design of the device could be modified to improve its performance in the near future.
\end{abstract}

\section{Keywords}

Milling, Fragmenting, Crushing, Soil, Planetary Ball Mill, Visualization

\section{Introduction}

When a soil sample is examined at a research institute, the soil must be fragmented into fine-grained soil particles with a median size of less than $2 \mathrm{~mm}$ in diameter, obeying the industrial standard. Several methods to comminute soil have been developed: mortar, pestle, and sieve by hand; milling machines using 
rods, balls, hammers, and air jets together with soils in a jar; and crushers using jaws, gyrating rods, rollers, electricity, disks, and edge runners.

One difficulty in improving the performance of milling devices is that the fragmentation mechanism due to the interactions between a ball and a clod in a jar are not fully understood. Pazesh et al. (2017) studied the relationship between comminution and amorphisation of $\alpha$-latctose monohydrate particles during ball milling under different milling conditions using a planetary ball mill (PM100, RetschCo.) [1]. A jar in the mill rotates twice per revolution of the sun wheel in a horizontal plane. This motion produces a complex interaction between the frictional and impact forces. They found that the particle size reduces as an exponential function of time. To understand what happens in the jar, Inoue et al. (1999) and Sinnott et al. (2017) investigated the motions of balls in a jar rotating in the horizontal axis using numerical simulations [2] [3]. The trajectories were found to depend on the ratio of the centrifugal force to the gravity. Dey et al. (2013) studied the comminution of coal and iron materials in a hammer mill under different feed rates and rotor speeds [4].

Crushers use pressing, hitting, and grinding methods that impart normal and shear forces onto clods. Crushers are usually used in an open space in dry grinding industries, and dust arising from the machines cause environmental pollution. Wheeldon et al. (2015) investigated the comminution process in pendular roller mills [5]. In such a mill, targeted particles are broken in the gap between the grinding ring and a roller attached to the pendulum rod. The roller generates compressive and tangential forces at the gap. The force of the roller arises from the centrifugal force due to its rotational motion; therefore, the angular velocity is important when determining the forces. In the case where attrition is the dominant mode of breakage, Jankovic (2001) analyzed the media stress intensity of vertical stirred mills and Hasan et al. (2017) investigated the breakage function under several conditions to reduce the energy consumption [6] [7].

The performance of our device is sufficiently satisfactory compared to conventional machines; however, the fragmentation mechanism in the jar is complicated. Therefore, to obtain a guide for developing a future device with a better performance, what happens in the jar is observed and the magnitudes of the forces generated by this device are measured. The fragmentation sequence of the clods is observed via high-speed movies to investigate the destruction process of the clods. Components of the forces generated by the mill device are measured to check their magnitudes. Interestingly, the rods do not collide with the clods frequently. It is found that a clod abruptly fragments into small pieces within the lapse of a certain time after starting the device. This means that it is necessary to accumulate destructive energy to fragment soil rather than to impulsively supply large energies. Therefore, the occasional impacts of the rods placed in a jar with clods lead to the sudden fragmentation of the soil. This suggests that a better design may be found to improve the performance of the device in the near future. 


\section{The Developed Rod Mill}

A new type of rod mill (Japanese patent No. 5055524) was developed for use at research institutes, as shown in Figure 1. Its specifications are given in Table 1.

The operating procedure to obtain soil particles with a median size of less than $2 \mathrm{~mm}$ in diameter for analyzes is as follows.

1) The sampled clods and two rods are placed in a jar, as shown in Figure 2.

The jar is placed on a turntable, as shown in Figure 3. It is possible to set four jars on the turntable. Noise arising from the rods colliding in the jar is muted by an outside cover, and rotation noise from the gears is muffled by the lid of the machine.

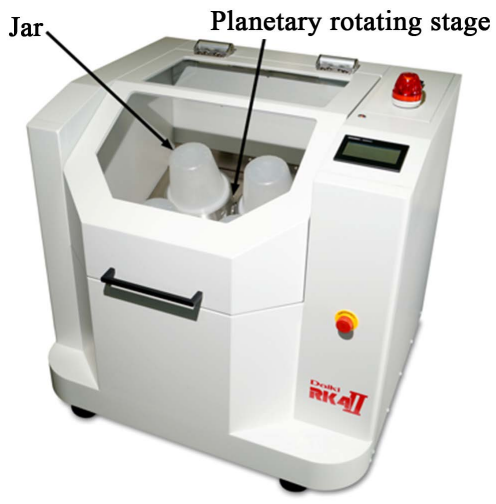

Figure 1. The developed rod mill (DIK-2610, Daiki Co.), which is free from soil dust and has a function of collection of soil particles with a median size of less than $2 \mathrm{~mm}$ in diameter.

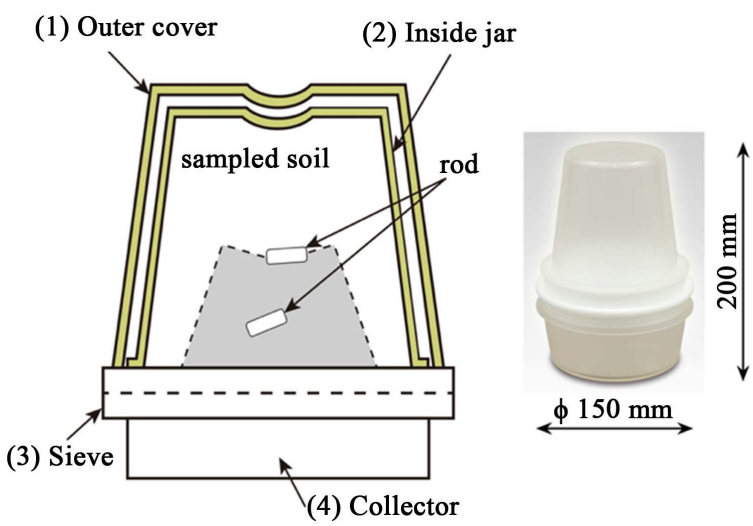

Figure 2. Schematic of the jar and its outward appearance.

Table 1. Specifications the rod mill.

\begin{tabular}{cccc}
\hline Size & W750 $\times$ D $670 \times \mathrm{F} 700 \mathrm{~mm}$ & Weight & $160 \mathrm{kgf}$ \\
\hline Electric voltage & $\mathrm{AC} 100 \mathrm{~V}(50 / 60 \mathrm{~Hz})$ & Driving time & $30-120 \mathrm{~s}$ \\
Electric current & Maximum $2.5 \mathrm{~A}$ & $\mathrm{RPM}$ & $100-800$ \\
Operating temperature & $20^{\circ} \mathrm{C}-30^{\circ} \mathrm{C}$ & & \\
Operating humidity & $30 \%-70 \% \mathrm{RH}$ & & \\
Rotation & Planetary gear & Speed ratio & $1:-4$ \\
\hline
\end{tabular}




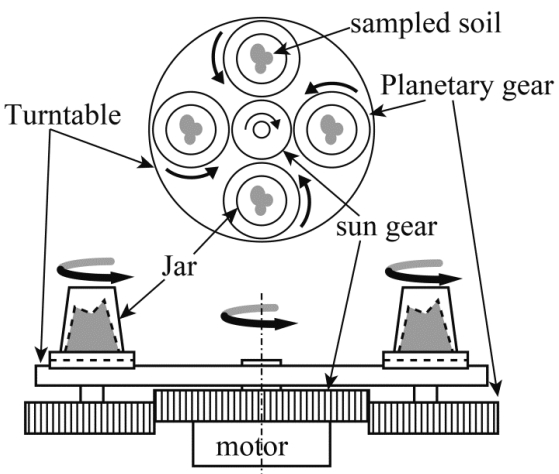

Figure 3. Turntable revolving in the clockwise direction using a sun gear and jars rotating in the counter-clockwise direction using a planetary worm gear (top view).

2) The jar is put on a turntable shown in Figure 3. It is possible to set four jars on the turntable. Noise arising from rods rampaging in a jar is shut out by an outside cover, and rotation noise from the gears is protected by a lid of the machine.

3) The soil particles pass through a sieve and fall into a collector cup, preventing soil dust from getting on the operator's hands, as well as keeping the working space clean.

4) The soil particles in the cup are transferred to an analyzing phase.

\section{Experimental Setup}

\subsection{Determination of the Operating Parameters}

For better performance, it is important to determine the necessary number and size of rods or balls because the rods directly interact with the sampled soil. Two types of examinations to determine the best number of rods and the optimum size of a pair of rods were performed to obtain the best performance during a two-minute period. The performance is expressed as the change in the amount of fragmented soil in $30 \mathrm{~s}, 60 \mathrm{~s}$, and $90 \mathrm{~s}$. The results are shown in Figure 4. The ordinate shows the amount of fragmented soil, and the abscissa shows the collected time (s). The diameter of the rod is $18 \mathrm{~mm}$. Different rod lengths of 38 $\mathrm{mm}, 45 \mathrm{~mm}, 50 \mathrm{~mm}$, and $70 \mathrm{~mm}$ were tested. Comparing the amounts of fragmented soil obtained using the different rod lengths at $30 \mathrm{~s}$ indicated that $L=45$ $\mathrm{mm}$ resulted in the largest amount of fragmented soil. This means that the fragmentation occurs earliest when a rod of $L=45 \mathrm{~mm}$ is placed in the jar. This rod is shown in Figure 5. It consists of a molybdenum cylinder and polypropylene case. Its weight is $0.53 \mathrm{~N}$.

The test results for the ratio of fragmentation using rods with different parameters after $60 \mathrm{~s}$ are shown in Figure 6. As seen in Figure 6, the fragmentation ratio increases when increasing the number of rods from one to four. To see the change in the performance for two rods, rods with different lengths were tested under different conditions. Comprehensively considering the results shown in Figure 6, two rods with $L=50 \mathrm{~mm}$ are sufficient to obtain the best performance. 


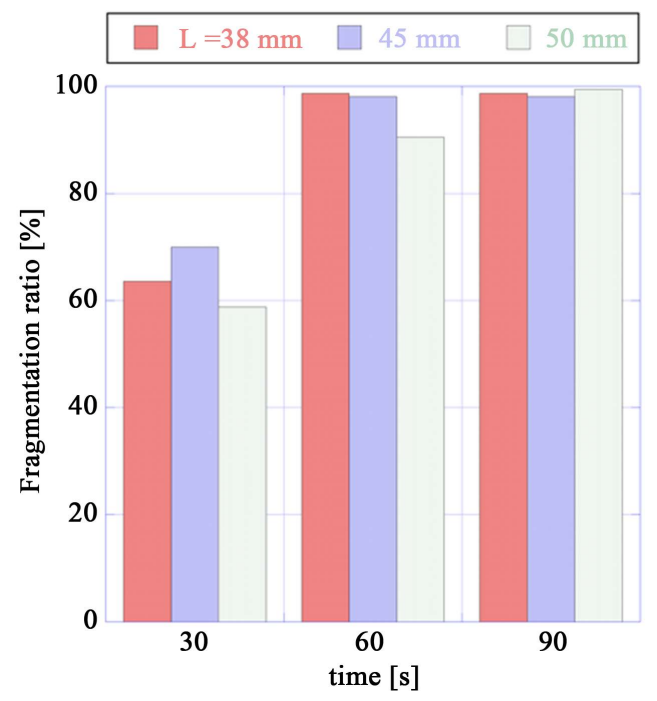

Figure 4. Ratios of fragmentation for different size rods.

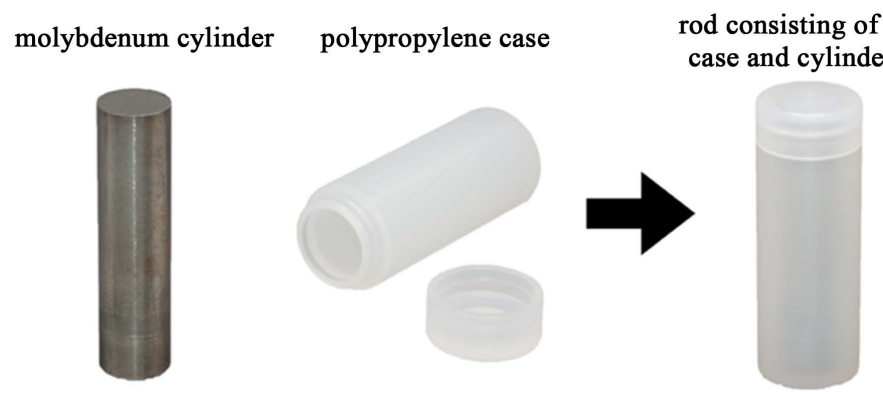

Figure 5. Molybdenum cylindrical rod is enclosed in a cylindrical polypropylene case to use the colliding in a jar.

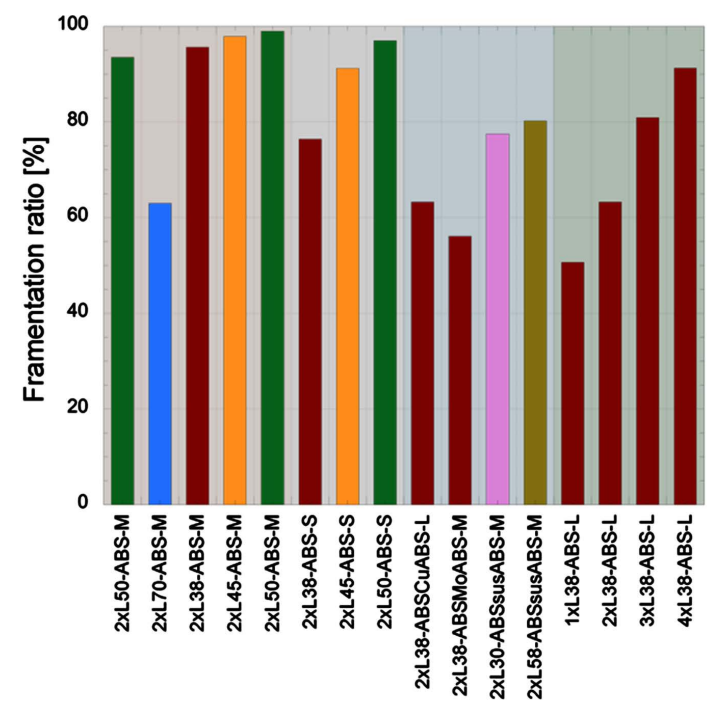

Exprtimental condition,

[Number of rods] $\mathrm{L}$ [Length of rod]-

[Material of rod]-[Size of soil shown as S, M, and L].

Figure 6. Ratio of fragmentation under different conditions after $60 \mathrm{~s}$. The same color of a bar in the chart shows the rod with the same length. The different background colors in the chart shows the different experimental conditions. 
A conventional mill requires many rods in the case of rod milling and needs to include many balls with different diameters in the case of ball milling. Therefore, the rod-to-soil ratio or the ball-to-soil ratio is an important parameter when determining the performance of a conventional machine. Conversely, only two shorter rods are required in this new method. This is a great advantage resulting in energy savings when driving the machine and allowing the jar to be downsized. In addition, this is convenient when cleaning the jar and rods for the next use.

The turning bed was tilted at an angle of $20^{\circ}$ following the result of an investigation of the fragmentation ratio at several tilting angles. Therefore, as seen in Figure 7, the clods and rods in the jar move along a complicated trajectory rather than in a horizontal plane because an additional rotating motion around the horizontal axis appears.

\subsection{Visualization of Fragmentation Process in the Jar}

To see what happens in the jar, movies of the clod and rod motions were taken using a compact high-speed CCD camera attached to the lid of the jar. The inside of the jar was illuminated by a LED light that was also attached to the jar. Therefore, the behavior of the contents of the jar can be observed in the fixed coordinates of the jar even though it rotates in a complicated manner. The loci of the clods and rods are observed as a result of their motion relative to the jar.

\subsection{Measurement of the Acceleration in the Jar}

The three components $a_{x}, a_{y}$, and $a_{z}$ in the $x^{-}, y^{-}$, and $z$-directions, respectively, of the forces, are measured using a tiny accelerometer (AccStick6, SysCom Co.) installed in the corner of the jar. Here, $\mathrm{x}$ indicates the circumferential direction, $\mathrm{y}$ indicates the radial direction, and $\mathrm{z}$ indicates the vertical direction.

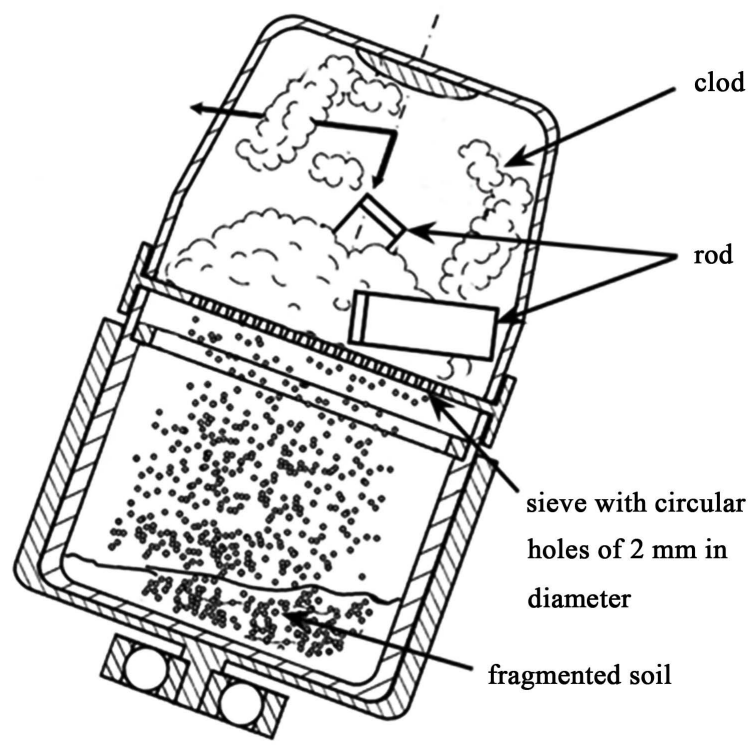

Figure 7. Motion of the colds and rods in the jar. 


\subsection{Measurement of the Strain Rate of a Clod via a Compression Device}

Understanding the magnitude of the force at the amorphous yield point is important to improve the machine because the magnitude of the force at that point shows the maximum force available for breaking the clod. The relationship of the force to the strain rate was obtained for several clods using a compression device (Instron 5566). Ten clod samples were compressed using an applied strain rate of $1 \mathrm{~mm} / \mathrm{min}$.

\section{Experimental Results}

\subsection{Visualization of Fragmentation Process in the Jar}

Figure 8 shows photos extracted every $10 \mathrm{~s}$ until $60 \mathrm{~s}$ from a movie of the fragmentation process in a jar. The initial amount of soil is $50 \mathrm{gf}$. Until at least $10 \mathrm{~s}$, the rods rotate together with the clods of soil. They do not appear likely to collide with each other because they move together at the same distance from each other. The view suddenly becomes clouded once the driving time is over $10 \mathrm{~s}$, as seen in the photo at $20 \mathrm{~s}$. That is because the fine dust of the soil is exfoliated from the surface via attrition. The number of clods decreases from $30 \mathrm{~s}$ to $40 \mathrm{~s}$; therefore, the fragmentation process proceeds abruptly during this period. Most clods disappear after $40 \mathrm{~s}$. Contrary to expectations, events where the rods crush the clods and to grind them into small pieces are not apparent; however, these events must occur because all the clods disappear, as seen in the photo at $50 \mathrm{~s}$. To see what happens during this period, a crushing event is observed in a situation with a small number of clods because, in this case, the view is clear of fine soil dust.

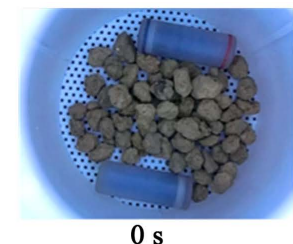

$0 \mathrm{~s}$

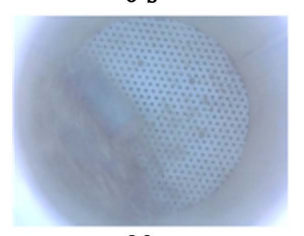

$30 \mathrm{~s}$

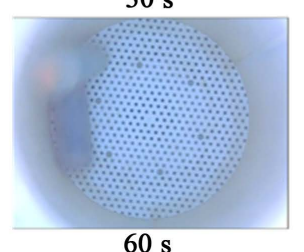

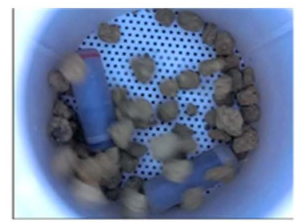

$10 \mathrm{~s}$

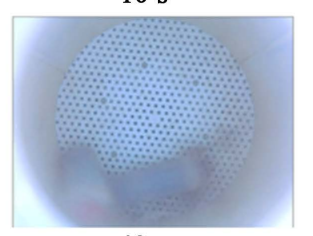

$40 \mathrm{~s}$

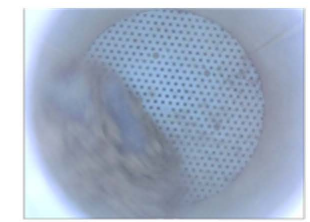

$20 \mathrm{~s}$

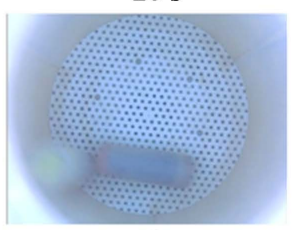

$50 \mathrm{~s}$

Figure 8. Visualization of the fragmentation process during a period of $60 \mathrm{~s}$. The fragmentation process in a jar is visualized. Initially located test soil fragmented to the soil particles with the rods. The soil particles pass through a sieve and fall into a collector cup. 
Figure 9 shows a crushing event arising due to a rod colliding with a clod. The rim, not the base, of the rod collides with a clod, and the clod breaks into two pieces. In this case, the breaking pattern of the crushing occurs due to compressive stress, not impacting stress. When the rim of the rod presses against the clod, its area is narrow and the pressure is concentrated on the surface of the clod. Therefore, the clod cracks at that point and breaks into two pieces. Contrary to crushing by compression, in the case of crushing by impact compression, clods fragment into small pieces. The relative velocity of the rods to the clods is small because the rods move together with the group of clods. However, the momentum of a rod is much larger than that of a clod even if their relative velocity is small because the weight ratio of a rod to a clod is approximately 50 in this experiment.

An example of crushing events due to hitting a clod with a rod is shown in Figure 10. The red line shows the locus of the clod marked by the red points during one rotation of the turntable. The locus is not a simple circle. This means that an unsteady centrifugal force, which is a function of the location of the clod in the jar, acts on the clod. Therefore, each clod experiences different forces because they move on different paths. They always experience unsteady forces from different directions even if rods and balls are not placed in the jar.

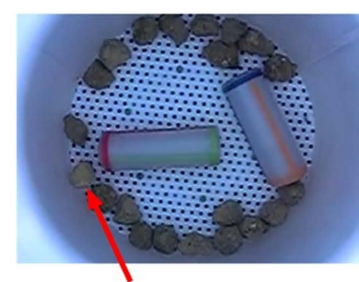

Just before collision

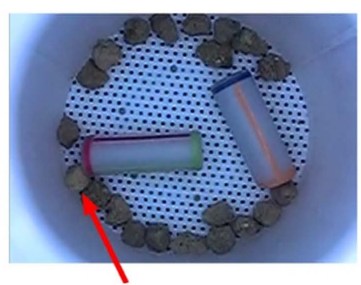

At an instant of collision

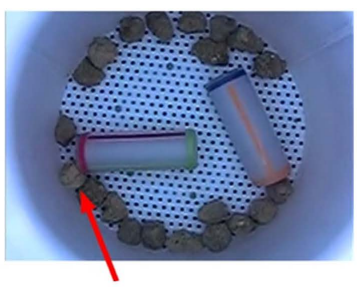

Breakage into two pieces

Figure 9. Crushing event of a rod colliding with a clod.

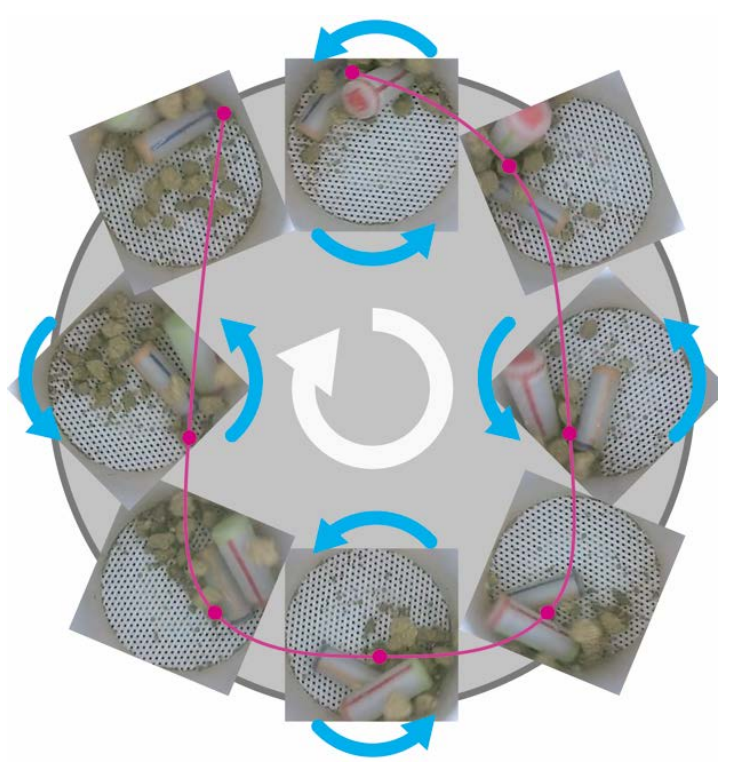

Figure 10. An example of crushing events of rods and clods. 
Three causes of comminution were reviewed by Little et al. (2016) to classify events extracted from previous studies: crushing via compressive stresses, impacts via collision, and attrition via shear stresses [7]. Hasan et al. (2017) indicate that these causes of comminution never take place alone in a milling machine [8]. There is the possibility of the clods breaking due to the unsteady forces described above if they exceed the amorphous yield point. However, clods never break into small pieces without the presence of rods. Therefore, it is thought that the collisions with the rod must trigger the catastrophic fragmentation of the clods into small pieces. The shape of the separated clods due to collisions with a rod appears to be the same shape as clods separated via the compressive stresses considered in Figure 9 and Figure 10. Therefore, catastrophic fragmentation is suspected to occur after the clods break into pieces.

\subsection{Measurement of the Acceleration in the Jar}

An example of waveforms of the acceleration generated in the jar is shown in Figure 11. The change in the magnitude of $a_{x}$ is from $-4.0 \mathrm{~g}$ to $+3.6 \mathrm{~g}$, that of $a_{y}$ is from $-7.3 \mathrm{~g}$ to $+0.1 \mathrm{~g}$, and that of $a_{\mathrm{z}}$ is from $+0.8 \mathrm{~g}$ to $+1.4 \mathrm{~g}$. Here, $\mathrm{g}$ is the gravitational acceleration. Therefore, the magnitudes of the tangential force along the sidewall and the compression force to the sidewall have the same order of 7 g. Converting this to the force acting on a clod, we find $1 \times 10^{-3} \times 7 \mathrm{~g} \times \sqrt{2}=98 \times$ $10^{-3} \mathrm{~N}$ because the mass of the clod is $1 \times 10^{-3} \mathrm{~kg}$. The force of a rod whose mass is $0.05 \mathrm{~kg}$ is $4.8 \mathrm{~N}$ according to the calculation shown above.

\subsection{Measurement of the Strain Rate of a Clod via a Compression Device}

The relationship of the force to the strain rate was obtained for several clods using the Instron 5566, as shown in Figure 12. Ten samples are performed. The samples are randomly picked up from the test soil. Different samples show different waveforms. A common feature of these samples is that the first peak

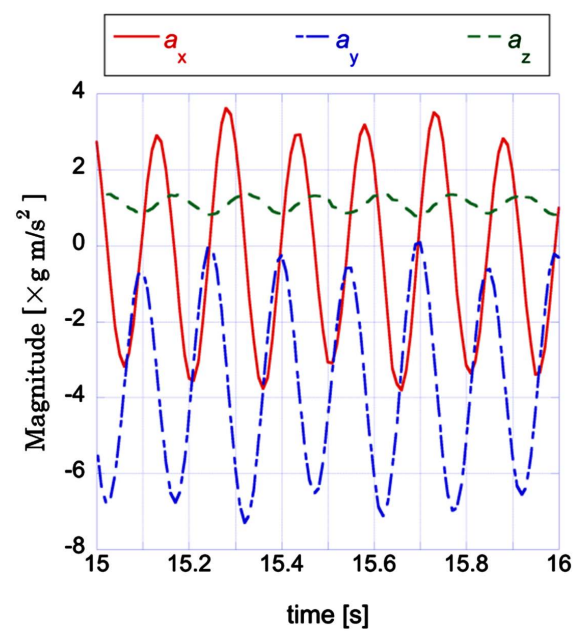

Figure 11. Waveform of the acceleration components obtained in a jar while driving the machine. 


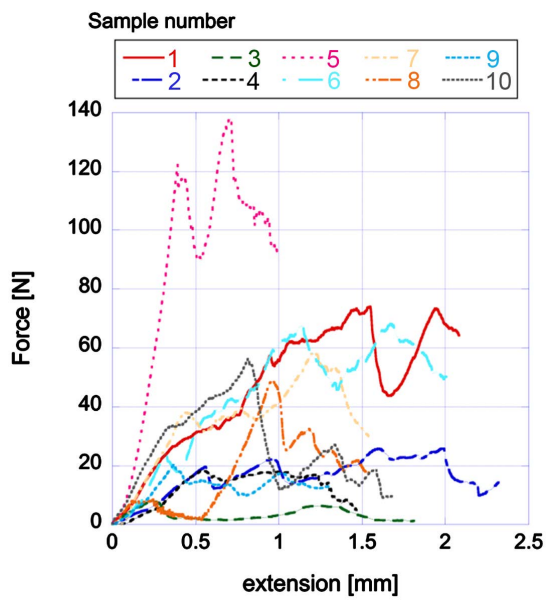

Figure 12. Changes in the force during the breakage process of a clod.

shows chipping, the second peak gives the amorphous yield point, and, after the second peak, the clod breaks into pieces. The magnitude at the second peak shows the maximum force. The average value of these maxima was $51.6 \mathrm{~N}$. This is 527 times larger than the force added to a clod via the centrifugal force. This is why clods never break when they are placed in the jar without a rod or ball. Moreover, the average maximum value is ten times larger than the centrifugal force given by a rod. This indicates the possibility that an impact compression event may exceed the observed maximum force.

\section{Conclusion}

We developed a planetary-type rod mill that can improve the comminution working efficiency, prevent the generation of soil dust, and make it easy to clean jars. Clods are broken by this device into fine-grained soil particles with a size of less than $2 \mathrm{~mm}$ in diameter within $2 \mathrm{~min}$. One objective of this study was to evaluate the performance of the mill by observing the fragmentation process of the clods. The process was visualized experimentally using a high-speed camera installed in a rotating jar in the device. The breakage of clods in the rod mill was considered morphologically according to the crushing patterns obtained by previous studies. The rod moves together with the clods in the jar. Therefore, violent collisions are not thought to be a major cause of clod breakage. The surfaces of the clods are first scraped and then appear to become fragile. However, if there are no rods in the jar, the clods never break into small pieces. Therefore, it is thought that the collisions of the rods likely trigger the catastrophic fragmentation of the clods into small pieces. The scenario of the fragmentation process is as follows. The attrition by shear stresses appears to be the first stage of comminution in this study because the view of the movie becomes cloudy due to the fine soil dust. The dust rises from the surfaces of the clods because the clod surfaces are exposed to abrasion. The angular surfaces of the clods are first scraped and then appear to become fragile because they are released from the surface binding force. There exist many chances for the rods to collide with the clods 
because they move closely together. As a result of preliminary experiments, three ridges were placed on the inside wall of the jar to add irregularities to the rod motion. Therefore, the irregular motion of the rods increases the chance of collision with the clods. The compression or impact forces due to the rods trigger the clods to fragment into small pieces. This suggests that the design of the device could be improved to further improve its performance in the near future.

\section{Conflicts of Interest}

The authors declare no conflicts of interest regarding the publication of this paper.

\section{References}

[1] Pazesh, S., Grasjo, J., Berggren, J. and Alderborn, G., (2017) Comminution-Amorphisation Relationships during Ball Milling of Lactose at Different Milling Conditions. International Journal of Pharmaceutics, 528, 215-227. https://doi.org/10.1016/j.ijpharm.2017.05.043

[2] Inoue, Y., Yokoyama, T., Tanaka, T. and Tsuji, Y. (1999) Three-Dimensional DEM Analysis of Collision Force in a Tumbling Ball Mill. Transactions of the Japan Society of Mechanical Engineers, Series $C, 65,1-7$. (In Japanese) https://doi.org/10.1299/kikaic.65.1

[3] Sinnott, M.D., Cleary, P.W. and Morrison, R.D. (2017) Combined DEM and SPH Simulation of Overflow Ball Mill Discharge and Trommel Flow. Minerals Engineering, 108, 93-108. https://doi.org/10.1016/j.mineng.2017.01.016

[4] Dey, S.K., Dey, S. and Das, A. (2013) Comminution Features in an Impact Hammer Mill. Powder Technology, 235, 914-920.

https://doi.org/10.1016/j.powtec.2012.12.003

[5] Wheeldon, M., Galk, J. and Wirth, K.E. (2015) Investigation of the Comminution Process in Pendular Roller Mills. International Journal of Mineral Processing, 136, 26-31. https://doi.org/10.1016/j.minpro.2014.09.013

[6] Jankovic, A. (2001) Media Stress Intensity Analysis for Vertical Stirred Mills. Minerals Engineering, 14, 1177-1186. https://doi.org/10.1016/S0892-6875(01)00135-2

[7] Little, L., Mainza, A.N., Becker, M. and Wiese, J.G. (2016) Using Mineralogical and Particle Shape Analysis to Investigate Enhanced Mineral Liberation through Phase Boundary Fracture. Powder Technology, 301, 794-804. https://doi.org/10.1016/j.powtec.2016.06.052

[8] Hasan, M., Palaniandy, S., Hilden, M. and Powell, M. (2017) Calculating Breakage Parameters of a Batch Vertical Stirred Mill. Minerals Engineering, 111, 229-237. https://doi.org/10.1016/j.mineng.2017.06.024 